\title{
The Quantum Cheshire Cat Effect in the Presence of Decoherence
}

\author{
Monika Richter, ${ }^{1}$ Bartosz Dziewit, ${ }^{1}$ and Jerzy Dajka $\mathbb{D}^{1,2}$ \\ ${ }^{1}$ Institute of Physics, University of Silesia in Katowice, Katowice, Poland \\ ${ }^{2}$ Silesian Center for Education and Interdisciplinary Research, University of Silesia in Katowice, Chorzów, Poland
}

Correspondence should be addressed to Jerzy Dajka; jerzy.dajka@us.edu.pl

Received 10 November 2017; Revised 12 March 2018; Accepted 28 March 2018; Published 9 May 2018

Academic Editor: Stephen C. Anco

Copyright (c) 2018 Monika Richter et al. This is an open access article distributed under the Creative Commons Attribution License, which permits unrestricted use, distribution, and reproduction in any medium, provided the original work is properly cited.

Even the subtle and apparently strange quantum effects can sometimes survive otherwise lethal influence of an omnipresent decoherence. We show that an archetypal quantum Cheshire Cat, a paradox of a separation between a position of a quantum particle, a photon, and its internal property, the polarization, in a two-path Mach-Zehnder setting, is robust to decoherence caused by a bosonic infinite bath locally coupled to the polarization of a photon. Decoherence affects either the cat or its grin depending on which of the two paths is noisy. For a pure decoherence, in an absence of photon-environment energy exchange, we provide exact results for weak values of the photon position and polarization indicating that the information loss affects the quantum Cheshire Cat only qualitatively and the paradox survives. We show that it is also the case beyond the pure decoherence for a small rate of dissipation.

\section{Introduction}

Counterintuitive world of quanta is a stage of various phenomena which are sometimes classified as paradoxes [1]. Their paradoxial character very often originates from a difference between mathematical modelling required for macro and micro scale or from a very special character and role of the quantum measurement. For a long time it has been expected that because of an omnipresent decoherence being (one of possible) mechanisms for a quantum-to-classical passage [2] quantum paradoxes are hardly present in reality. Nowadays we know that it is not the case; many applications of entanglement can serve as spectacular examples of observing and utilizing essentially quantum properties. This is why it is so important for a given "paradox" to answer a natural question: does it remain "paradoxial" also in the presence of decoherence?

In this work we focus on the quantum Cheshire Cat, an effect recently added to a list of quantum "paradoxes" [3], which has attracted considerable interest of both theoretical and experimental physicists [4-6]. This is a paradox of separation of two properties of a quantum particle named in an analogy to the behaviour of the Cheshire Cat and its grin, a character in the novel Alice's Adventures in Wonderland by Lewis Carroll. Alice, who (before she fell down in the Rabbit Hole) "has often seen a cat without a grin but never a grin without a cat," would have been surprised seeing a photon, a cat, separated from its polarization, a grin.

The archetype of the quantum Cheshire Cat paradox proposed in [3] is a two-path Mach-Zehnder-like setting for a photon with an internal degree of freedom, the polarization. A state space of the considered system is a 4-dimensional Hilbert space $\mathscr{H}=\mathscr{C}^{2} \otimes \mathscr{C}^{2}=\operatorname{span}\{|L\rangle,|R\rangle\} \otimes \operatorname{span}\{|H\rangle,|V\rangle\}$, where $L, R$ and $H, V$ label the path "chosen" by the photon (either left $L$ or right $R$ ) and its polarization (horizontal $H$ or vertical $V$ ), respectively. The photon is prepared (preselected) in a state

$$
|\Psi\rangle=\frac{1}{\sqrt{2}}(i|L H\rangle+|R H\rangle)
$$

and then detected (postselected) in a state

$$
|\Phi\rangle=\frac{1}{\sqrt{2}}(|L H\rangle+|R V\rangle) .
$$


Identification of the photon in one of the possible paths (arms) corresponds to a measurement related to the projectors:

$$
\begin{aligned}
& \Pi_{L}=|L\rangle\langle L|, \\
& \Pi_{R}=|R\rangle\langle R|,
\end{aligned}
$$

whereas a measurement of its polarization in a given (either left or right) arm requires the projectors

$$
\begin{gathered}
\sigma_{L}=\Pi_{L} \sigma_{z}, \\
\sigma_{R}=\Pi_{R} \sigma_{z},
\end{gathered}
$$

where $\sigma_{z}=|+\rangle\langle+|-|-\rangle\langle-|$ for $| \pm\rangle=[|H\rangle \pm i|V\rangle] / \sqrt{2}$. As detection of the Cheshire Cat requires simultaneous measurement for both paths of the photon, one applies there the weak measurement scheme [3,7]. Both the interpretations and the broad possible applications of the quantum weak values are presented in [7]. Here, following [7], we simply define the $n$th order weak value related to an observable $X$ as

$$
\langle X\rangle_{w}^{n}=\frac{\left\langle\Phi\left|X^{n}\right| \Psi\right\rangle}{\langle\Phi \mid \Psi\rangle} .
$$

There is a natural interpretation of the weak value if one considers unitary transformation $U=\exp (-i \epsilon X)$ generated by an observable $X$. The quantity $\langle f|U| i\rangle$ is a related transition amplitude from the preselected initial state $|i\rangle$ into the final $|f\rangle$ which can be then postselected. For sufficiently small $\epsilon$ one can Taylor-expand the amplitude with the expansion terms containing the weak values in (5) up to some finite order related to the magnitude of $\epsilon$.

In the weak interaction regime, one can neglect higher order terms; that is, one can limit to $\langle f|U| i\rangle \approx\langle f|(1-i \epsilon X)| i\rangle$. As the most of the experiments performed so far operate in this regime, further we limit to the first-order case $n=1$ and utilize the basic interpretation of $\langle X\rangle_{w} \equiv\langle X\rangle_{w}^{1}$ as a change of the detection probability $|\langle\Phi \mid \Psi\rangle|$ in the presence of (weak) interaction generated by $X$. Let us remember that generically the weak value of an observable is a complex, yet measurable [7], quantity. For the quantum Cheshire Cat experiment, with given pre- (see (1)) and postselected (see (2)) states, one obtains [3]

$$
\begin{aligned}
& \left\langle\Pi_{L}\right\rangle_{w}=1, \\
& \left\langle\Pi_{R}\right\rangle_{w}=0
\end{aligned}
$$

for "the cat" and

$$
\begin{aligned}
& \left\langle\sigma_{L}\right\rangle_{w}=0, \\
& \left\langle\sigma_{R}\right\rangle_{w}=1
\end{aligned}
$$

for its "grin," respectively. According to the interpretation proposed in [3] and originating from Alice in Wonderland one obtains the cat residing in the left path separated from its grin which appears in the right path. Let us emphasize that our primary aim is to present that the Cheshire Cat effect, in its archetype formulated using quantum weak values, survives in the presence of decoherence of a certain, relatively general, type. Discussion of controversies concerning this effect such as those reported in $[4,8,9]$ or attempts to extend our results to different treatments or formalism is beyond the assumed scope of our work.

The paper is organized as follows: (i) we present qualitative considerations showing how the predictions of [3] become modified by decoherence caused by an environment locally coupled to a photonic polarization. (ii) Further we exemplify our model and apply it to a simplest case of pure decoherence when one can neglect a photon-environment energy exchange. (iii) Our next step is to include dissipation. We are going to allow for a weak energy transfer between our system and its environment.

\section{The Cat, Its Grin, and the Noise}

In this section, we discuss a general model of the quantum Cheshire Cat in the presence of decoherence caused by an environment coupled locally to the polarization of the photon. To formalize our discussion, we expand the state space into the triple $\mathscr{H}=\mathscr{C}^{2} \otimes \mathscr{C}^{2} \otimes \mathscr{H}_{E}$, where the last term corresponds to an environment $E$ which needs to be included and which is going to be specified in the next section. We limit our attention to the simplest setting of a noisy preselection and we assume that the environment $E$ couples to photonic polarization only locally, that is, only in one of the two arms of the interferometer corresponding to either the sector $L$ or $R$ of the state space $\mathscr{H}$. We also assume that initially, prior to any interaction, the environment is in a pure state $|\Omega\rangle \in \mathscr{H}_{E}$. In such a case there are two possible "noisy" preselected states: the first, where the polarization is affected by $E$ in a right path

$$
\left|\Psi_{R}\right\rangle=\frac{1}{\sqrt{2}}\left(i\left|L H_{0}\right\rangle+\left|R H_{t}\right\rangle\right)
$$

and the second, for $E$ affecting polarization in the left path,

$$
\left|\Psi_{L}\right\rangle=\frac{1}{\sqrt{2}}\left(i\left|L H_{t}\right\rangle+\left|R H_{0}\right\rangle\right)
$$

The time $t$-parameterized family of states $\left|H_{t}\right\rangle=U(t)|H\rangle|\Omega\rangle$ results from the polarization-environment interaction after a time $t$. A unitary operator $U(t)$, such that $U(0)=\mathscr{I}$ (the identity operator), describes an interaction between polarization and the environment. We assume that the postselection is not affected by the presence of the environment; that is,

$$
|\Phi\rangle=\frac{1}{\sqrt{2}}\left(\left|L H_{0}\right\rangle+\left|R V_{0}\right\rangle\right)
$$

However, the weak values which are a figure of merit for the Cheshire Cat paradox become modified by decoherence

$$
\langle X\rangle_{w}^{Q}=\frac{\left\langle\Phi|X| \Psi_{Q}\right\rangle}{N_{Q}}, \quad Q=L, R .
$$


The normalization factors read as follows:

$$
\begin{aligned}
& N_{R}=\left\langle\Phi \mid \Psi_{R}\right\rangle=\frac{1}{2}\left(i+\left\langle V_{0} \mid H_{t}\right\rangle\right) \\
& N_{L}=\left\langle\Phi \mid \Psi_{L}\right\rangle=\frac{i}{2}\left\langle H_{0} \mid H_{t}\right\rangle .
\end{aligned}
$$

The weak value in (11) quantifies a weakly measured quantity of a physical system coupled to an environment. For the preselection given in (8) (indicated by the superscript $R$ below) the weak value of "the cat" position is

$$
\begin{aligned}
\left\langle\Pi_{L}\right\rangle_{w}^{R} & =\frac{i}{2 N_{R}}, \\
\left\langle\Pi_{R}\right\rangle_{w}^{R} & =\frac{\left\langle V_{0} \mid H_{t}\right\rangle}{2 N_{R}}=\frac{\left\langle V_{0} \mid H_{t}\right\rangle}{i+\left\langle V_{0} \mid H_{t}\right\rangle},
\end{aligned}
$$

whereas for $\sigma_{L, R}$ in (4) "the grin" is

$$
\begin{aligned}
\left\langle\sigma_{L}\right\rangle_{w}^{R} & =0, \\
\left\langle\sigma_{R}\right\rangle_{w}^{R} & =\frac{\left\langle V_{0}\left|\sigma_{z}\right| H_{t}\right\rangle}{2 N_{R}} .
\end{aligned}
$$

Let us notice that for the preselection in (8) decoherence affects the cat, originally residing in the (noiseless) $L$-sector whereas the grin is solely confined to the $R$-sector of the system. It is in an apparent contrast to what occurs for the preselection in (9). In that case (indicated by the superscript $L$ below), corresponding to a noisy $L$-sector, originally occupied by the cat, the cat's position remains confined to the $L$ sector of the system

$$
\begin{aligned}
\left\langle\Pi_{L}\right\rangle_{w}^{L} & =\frac{i\left\langle H_{0} \mid H_{t}\right\rangle}{2 N_{L}} \equiv 1, \\
\left\langle\Pi_{R}\right\rangle_{w}^{L} & =0
\end{aligned}
$$

whereas the grin becomes wiped off by decoherence

$$
\begin{aligned}
\left\langle\sigma_{L}\right\rangle_{w}^{L} & =\frac{i\left\langle H_{0}\left|\sigma_{z}\right| H_{t}\right\rangle}{2 N_{L}}, \\
\left\langle\sigma_{R}\right\rangle_{w}^{L} & =\frac{i}{2 N_{R}} ;
\end{aligned}
$$

that is, it appears in both $R$ - and $L$-sectors of the system. One can say that for the preselection in (8) decoherence attracts the cat whereas for the preselection in (9) decoherence attracts the grin.

The original quantum Cheshire Cat originates from a very peculiar asymmetry between initial preparation (preselection) of the system having internal degree of freedom and its postselection via a very specific measurement scheme. In our considerations, instead of studying more realistic schemes presented, for example, in [6], we limit our considerations to the archetype proposal schematically presented in Figure 1 of [3]. However, let us notice that an effect of decoherence in (9) and (8) is fully general as it incorporates a well established system-environment modelling [10] with a unitary evolution $U(t)$ encoding both the time evolution of the system and the bath and its interaction which is going to be specified in the following part of the paper. The only thing assumed so far is (i) that the environment couples to the internal degree of freedom (the polarization) only and (ii) that the environment is local; that is, it is present in one of two arms of the interferometer only. Locality of the decoherence is justified since there is a spatial separation between photonic paths in the interferometer in particular if one limits to the polarization-environment interaction $[11,12]$.

The result is partial neutralization of the asymmetry between the preselected and the postselected state of the system. Let us also notice that due to the asymmetry the Cheshire Cat effect survives also in the presence of decoherence in an apparent contrast to some other quantum effects for which decherence can be lethal. Here entanglement serves as a natural example [13]. In particular, the "smile" in the left arm of the interferometer never occurs provided that the "noise" affects only the right arm (cf. $\left\langle\sigma_{L}\right\rangle_{w}^{R}=0$ in (14)) related to the preselection in (8), which is not the case if the decoherence affects the lest arm in (16). Formally, it is due to the effect of $\Pi_{L}$ projection present in (4). More physically, one interprets it as a "conditional measurement" with a condition selecting the subspace of states not affected by decoherence. Notice that it is not the case for the preselection in (9). Obviously, the general statements of this section became modified for a particular decoherence mechanism as presented below. However, for any particular choice satisfying the assumptions (i) and (ii), the effect can be quantitative at most.

\section{Pure Decoherence}

In this section, we exemplify our discussion using probably the simplest model of decoherence, the pure dephasing model $[10,14]$. In this model, one operates on the time scales such that one can neglect an energy exchange between the system and its environment [15]. In our case photons couple to the environment via their polarization. For the pure decoherence the system-environment Hamiltonian is assumed to have the particular form

$$
H=|+\rangle\left\langle+\left|\otimes H_{+}+\right|-\right\rangle\langle-| \otimes H_{-}
$$

indicating its block-diagonal structure which will be used for gaining exact results for time evolution of the decoherenceaffected preselected state. The block-diagonal structure arises from a very peculiar symmetry of the model. The pure decoherence is along quantization axis and hence it corresponds to transversal relaxation of polarization degree of freedom. We assume that initially the photonic polarization $|H\rangle,|V\rangle$ and initial states of the environment $|\Omega\rangle$ are separated and read as follows:

$$
\begin{aligned}
& \left|V_{0}\right\rangle=\frac{i}{\sqrt{2}}(|-\rangle-|+\rangle)|\Omega\rangle \\
& \left|H_{0}\right\rangle=\frac{1}{\sqrt{2}}(|-\rangle+|+\rangle)|\Omega\rangle .
\end{aligned}
$$




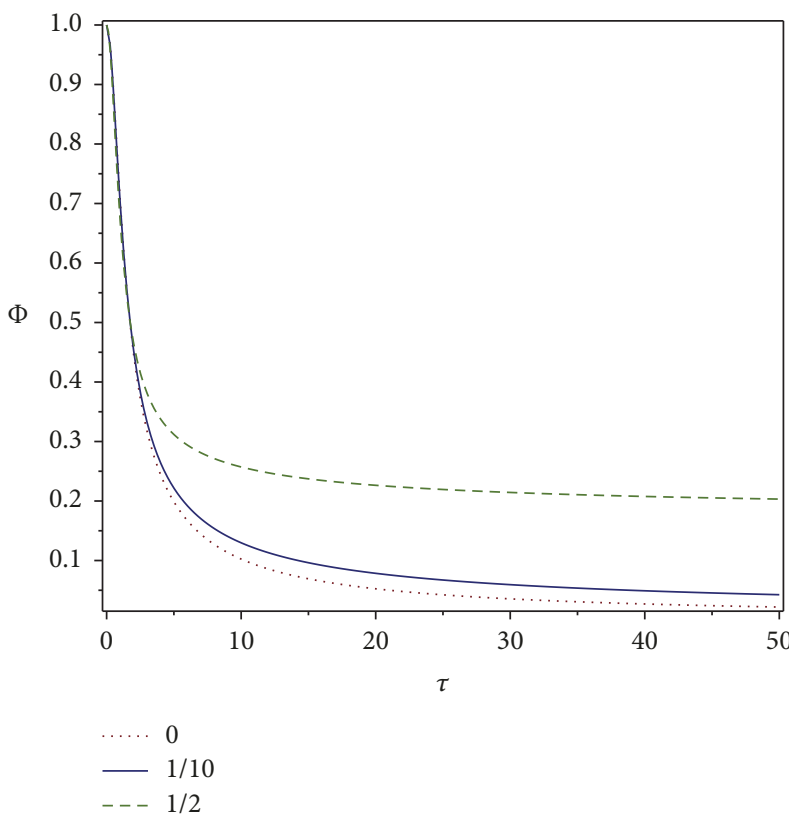

(a)

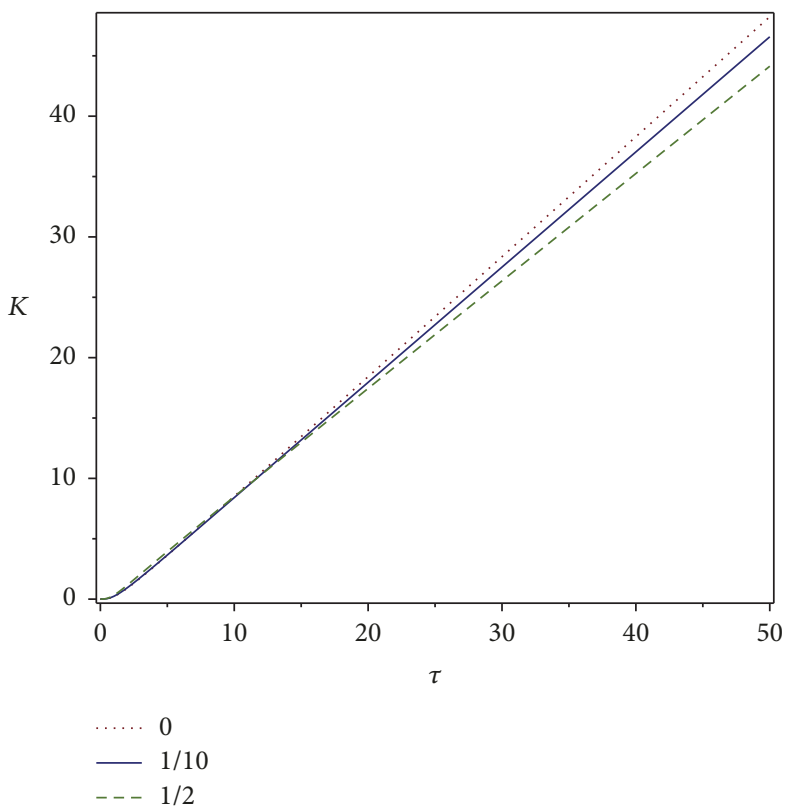

(b)

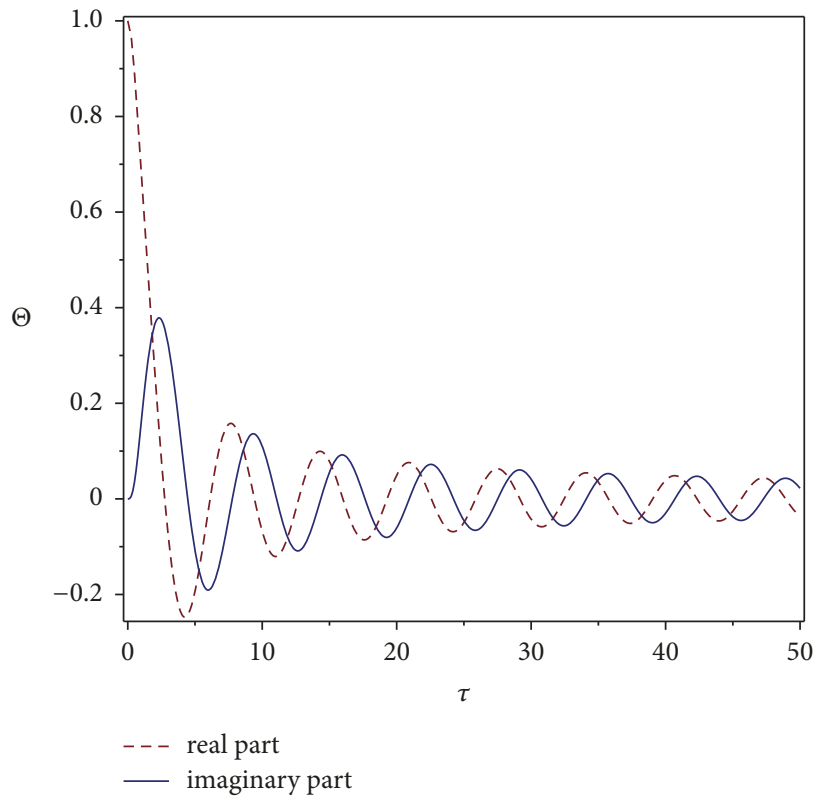

(c)

Figure 1: Plot of $\Phi=\Phi_{+}(t)(\mathrm{a})$ and $K=K_{+}(t)(\mathrm{b})$ in (29) in the presence of symmetric pure dephasing with $h(\omega)=\omega$ and for different values of $\mu=0,1 / 10,1 / 2$ and in (c) the real and imaginary part of $Q=Q_{+}$for $\mu=1 / 10$. The amplitudes $\lambda_{ \pm}=1$ and time $\tau$ is in units $100 / \omega_{c}$.

For the preselection defined in both (8) and (9), the horizontal polarization is modified by the decoherence. For the pure decoherence generated by (17), one obtains

$$
\left|H_{t}\right\rangle=\frac{1}{\sqrt{2}}\left(|+\rangle\left|\Omega_{t}^{+}\right\rangle+|-\rangle\left|\Omega_{t}^{-}\right\rangle\right),
$$

where $\left|\Omega_{t}^{ \pm}\right\rangle=\exp \left(-i H_{ \pm} t\right)|\Omega\rangle$. An effect of (pure) decoherence on the Cheshire Cat paradox is fully governed by a quantity

$$
Q_{ \pm}=\left\langle\Omega \mid \Omega_{t}^{+}\right\rangle \pm\left\langle\Omega \mid \Omega_{t}^{-}\right\rangle
$$

which is an overlap sum/difference between the initial and time-evolving states of the environment. The weak values for the position of the photon calculated for the "noise-affected" preselection in (8) read as follows:

$$
\begin{aligned}
\left\langle\Pi_{L}\right\rangle_{w}^{R} & =\frac{1}{1+Q_{-} / 2}, \\
\left\langle\Pi_{R}\right\rangle_{w}^{R} & =\frac{Q_{-}}{2\left(1+Q_{-} / 2\right)} .
\end{aligned}
$$


The "grin of the cat" corresponding to (8), that is, the weak values of the photonic polarization, is given by

$$
\begin{aligned}
\left\langle\sigma_{L}\right\rangle_{w}^{R} & =0, \\
\left\langle\sigma_{R}\right\rangle_{w}^{R} & =\frac{Q_{+}}{2\left(1+Q_{-} / 2\right)} .
\end{aligned}
$$

The preselection in (8) describes the case when the environment couples to the right path (the $R$-sector) of the interferometer, the one occupied by the grin. Let us notice that despite an apparent modification of the position of the cat in (21) the effect on the cat's grin in (22) is only qualitative in comparison to the noiseless case in (7). It is in contrast to the case of the preselection in (9) when the environment couples to the $L$-sector of the interferometer. Such a decoherence does not modify the cat's position:

$$
\begin{aligned}
\left\langle\Pi_{L}\right\rangle_{w}^{L} & =1, \\
\left\langle\Pi_{R}\right\rangle_{w}^{L} & =0,
\end{aligned}
$$

but rather the grin which now appears also in the left path, that is, in the $L$-sector:

$$
\begin{aligned}
\left\langle\sigma_{L}\right\rangle_{w}^{L} & =\frac{Q_{-}}{Q_{+}}, \\
\left\langle\sigma_{R}\right\rangle_{w}^{L} & =\frac{2}{\left(2+Q_{-}\right)} .
\end{aligned}
$$

It is in accordance with our previous conclusion that the decoherence attracts the cat for the preselection in (8) and the grin for (9), respectively.

Typically, pure decoherence or dephasing models $[10,16]$ are described by a linear coupling of a system to a bosonic bath:

$$
\begin{aligned}
H_{ \pm}= & \int_{0}^{\infty} d \omega h(\omega) a^{\dagger}(\omega) a(\omega) \\
& \pm \int_{0}^{\infty} d \omega h(\omega) g_{ \pm}(\omega)\left(a^{\dagger}(\omega)+a(\omega)\right),
\end{aligned}
$$

where $a(\omega)$ and $a^{\dagger}(\omega)$ generate the Heisenberg-Weyl algebra $[17,18]$. Let us notice that for the most natural symmetric dephasing $g_{+}(\omega)=g_{-}(\omega)$ one clearly obtains $Q_{-}=0$ and $Q_{+}=Q$ for some (generically) complex quantity $Q=\langle\Omega|$ $\left.\Omega_{t}^{+}\right\rangle$(which is going to be determined below). This property follows strictly from the symmetry of the model in (25) and depends on neither the coupling $g(\omega)$ nor the spectral properties of the bath $h(\omega)$. For the symmetric case and the preselections $\left|\Psi_{L, R}\right\rangle$, in (8) and (9), the weak values of the cat's position and grin in the $L$-path of the interferometer are not affected by decoherence

$$
\begin{gathered}
\left\langle\Pi_{L}\right\rangle_{w}^{L, R}=1 \\
\left\langle\Pi_{R}\right\rangle_{w}^{L, R}=0 \\
\left\langle\sigma_{L}\right\rangle_{w}^{L, R}=0
\end{gathered}
$$

whereas the grin in the $R$-sector

$$
\begin{aligned}
& \left\langle\sigma_{R}\right\rangle_{w}^{L}=\frac{2}{Q}, \\
& \left\langle\sigma_{R}\right\rangle^{R}=\frac{Q}{2}
\end{aligned}
$$

differs only quantitatively from the results of the noiseless archetype introduced in [3]. Utilizing the Heisenberg-Weyl symmetry of the Hamiltonian in (25), one can calculate

$$
\left|\Omega_{t}^{ \pm}\right\rangle=e^{i K_{ \pm}(t)} D\left( \pm \frac{g_{ \pm}(\omega)}{h(\omega)}\left(1-e^{-i h(\omega) t}\right)\right)|\Omega\rangle,
$$

where $K_{ \pm}(t)=\int_{0}^{\infty} d \omega\left(g_{ \pm}(\omega) / h(\omega)\right)^{2}(h(\omega) t-\sin (h(\omega) t))$ with the displacement operators $[17,18] D(f)=$ $\exp \left[\int_{0}^{\infty} d \omega\left(f(\omega) a^{\dagger}(\omega)-\right.\right.$ h.c. $\left.)\right]$ which span the HeisenbergWeyl algebra $D(q) D(f)=\exp \left[i \int_{0}^{\infty} d \omega q(\omega) \bar{f}(\omega)\right] D(f+q)$.

The Heisenberg-Weyl symmetry is of particular usefulness if it is possible to express quantum states of the environment in terms of coherent states $|f\rangle=D(f)|\Omega\rangle$ satisfying $\langle q \mid f\rangle=\exp \left[\int_{0}^{\infty} d \omega\left[\bar{q}(\omega) f(\omega)-(1 / 2)|q(\omega)|^{2}-\right.\right.$ $\left.\left.(1 / 2)|f(\omega)|^{2}\right]\right]$. Finally, applying the above properties of the displacement operators to (28) and assuming that $|\Omega\rangle$ is bosonic vacuum state (the ground state of the environment), one obtains

$$
Q_{ \pm}=e^{i K_{+}(t)} \Phi_{+} \pm e^{i K_{-}(t)} \Phi_{-}
$$

with $\Phi_{ \pm}=\exp \left[-\int_{0}^{\infty} d \omega\left(g_{ \pm}^{2}(\omega) / h^{2}(\omega)\right)[1-\cos (h(\omega) t)]\right]$. There is a particular and natural choice

$$
\begin{aligned}
g_{ \pm}^{2}(\omega) & =\lambda_{ \pm} \omega^{1+\mu} \exp \left(\frac{-\omega}{\omega_{c}}\right), \\
h(\omega) & =\omega
\end{aligned}
$$

motivated by a standard spectral density of bosonic environments [10] with the parameter $\mu$ which allows for classification of environments as sub-Ohmic $(\mu<0)$, Ohmic $(\mu=0)$, and super-Ohmic $(\mu>0)$. To avoid mathematical controversies [14], one can limit a discussion to strictly superOhmic environments, $\mu>0$. For the choice in (30), one can evaluate $Q_{ \pm}$exactly using Euler Gamma function (cf. [19]):

$$
\begin{aligned}
\mathscr{L}(\mu) & =\int_{0}^{\infty} d \omega \omega^{\mu-1} \exp \left(\frac{-\omega}{\omega_{c}}\right)=\Gamma(\mu) \omega_{c}^{\mu}, \\
\mathscr{M}(\mu, t) & =\int_{0}^{\infty} d \omega \omega^{\mu-1} \exp \left(\frac{-\omega}{\omega_{c}}\right) \cos (\omega t) \\
& =\mathscr{L}(\mu) \frac{\cos \left[\mu \arctan \left(\omega_{c} t\right)\right]}{\left(1+\omega_{c}^{2} t^{2}\right)^{\mu / 2}}, \\
\mathscr{N}(\mu, t) & =\int_{0}^{\infty} d \omega \omega^{\mu-1} \exp \left(\frac{-\omega}{\omega_{c}}\right) \sin (\omega t) \\
& =\mathscr{L}(\mu) \frac{\sin \left[\mu \arctan \left(\omega_{c} t\right)\right]}{\left(1+\omega_{c}^{2} t^{2}\right)^{\mu / 2}} .
\end{aligned}
$$


It follows from the discussion that the Cheshire Cat effect is preserved in symmetric pure dephasing environments. It is not the case if one allows for a certain degree of asymmetry. General dephasing models studied in various contexts in [1921] contain, as a special case, the symmetric van Hove model [22], with $g_{+}(\omega)=g_{-}(\omega)$, and the Friedrichs model [23], $g_{+}(\omega) \neq g_{-}(\omega)=0$. Let us consider an extreme Friedrichslike asymmetry, that is, (30) characterized by $\lambda_{+}=\lambda$ and $\lambda_{-}=0$ such that $Q_{-}=Q_{+}=Q / 2$. For the preselection $\left|\Psi_{R}\right\rangle$, the cat, up to now residing only in the $L$-sector, appears also in the right $R$ arm of the interferometer according to (21). At the same time, as one infers from (22), the grin remains confined in the right path of the interferometer. The situation for the preselection $\left|\Psi_{L}\right\rangle$ is complementary (cf. (23) and (24)): the cat remains confined to the left path but its grin is present in both $R$ - and $L$-paths.

Pure dephasing [14] belongs to a very narrow class of exact models of open quantum systems. Despite its simplicity, it can be effectively utilized in modelling of certain class of real systems [15]. One can quantify validity of a chosen model of decoherence via recognizing the dominant time scale in the system. There are at least three different time scales quantifying an open quantum system [24]: the first which is related to the typical separation of energy levels of a system, the second quantifying spectral properties of environment, and the third, related to energy of system-environment interaction. Roughly speaking, ordering of these time scales implies the type of approximation to be credibly used for describing reduced dynamics. Contrary to, for example, a weak coupling approximation, the pure dephasing model applied in this section is exact but for the price of credibility limited to the time scales which are short with respect to the time scales of energy dissipation [14]. There are real systems where such a condition is satisfied and which do fulfill pure depahsing conditions [15]. The parameter which quantifies decoherence in general and in particular the pure dephasing is $\mu$ in (30). It is known [14] that only superOhmic environments characterized by $\mu>0$ grant existence of a ground state of the total system. In other cases, due to the infrared problem, there is no ground state or even the spectrum of the total system can be unbounded from below.

Studying of the quantity $Q_{ \pm}$in (29) allows filling with a physical content formulas of the previous section where we present how an arbitrary local decohernce affects the Cheshire Cat. We plot the amplitude $\Phi \pm$ and the phase $K_{ \pm}$as a function of time in (a) and (b) of Figure 1. The range of time $\tau$ is related to the cut-off frequency $\omega_{c}$. The time scale of the Cheshire Cat experiment is given by a typical passage time in the interferometer and hence it is short. As a result, the long time asymptotic limit, despite its mathematical exactness, is of rather small usefulness for us. We focus on the short time properties and we infer that the time dependence of $K$ and an $\mu$-ordering of the $F$ graphs is very close to linear. The $\mu=0$ (Ohmic) case should be considered as a formal limit $\mu \rightarrow 0$ due to the above-mentioned mathematical troubles with an infrared divergence. The result, plotted in (c) of Figure 1, indicates the dominant role played by the real part of $Q$ in modifying the Cheshire Cat weak values which, physically, is governed by spectral properties of the environment hidden in $\mu$. This conclusion remains valid also in the case of asymmetric dephasing which, in a time scales shorter than a time scale of energy dissipation, approximates real optical systems with an anisotropic coupling to the polarization $[11,12]$.

\section{Dissipation}

Pure decoherence is obviously a significant simplification and a far-reaching but useful [15] idealization of generic systems exchanging energy with their surrounding. In this section, we take one step towards real system and assume that the photon coupled with bosonic bath via its polarization can dissipate, that is, exchange energy with the bath; that is, we allow for a longitudinal relaxation of photonic polarization. The previously discussed pure decoherence equipped with dissipation forms a general decoherence model with both transversal and longitudinal relaxation which, due to its complexity, which requires approximate treatment [10]. Here, similarly to [25], we assume that the rate of dissipation is small compared to other rates in the system; that is, the time evolution of the system is close to the pure dephasing discussed in the previous section. This assumption clearly bounds an applicability and credibility of our results to a relatively narrow class of dissipative systems. However, there is a significant benefit related to that assumption: the dissipation can be treated by means of perturbation theory [26, 27]. Here, following [25], we consider

$$
H_{\varepsilon}=H+\varepsilon[|+\rangle\langle-|+|-\rangle\langle+|],
$$

where $H$, given in (17) with $H_{+}$in (25) but with $g_{+}=g_{-}$, describes symmetric pure decoherence and the remaining part of (32) is related to tunneling between (circular) polarization + and - with a perturbatively small rate $\varepsilon \ll 1$. For $U_{ \pm}(t)=\exp \left(-i H_{ \pm} t\right)$ the (Schrödinger picture) first-order perturbative correction [27] to (19) is given by

$$
\left|H_{t}^{\varepsilon}\right\rangle=\frac{1}{\sqrt{2}}\left[|+\rangle\left|\Delta_{t}^{+}\right\rangle+|-\rangle\left|\Delta_{t}^{-}\right\rangle\right]
$$

where

$$
\begin{aligned}
& \left|\Delta_{t}^{+}\right\rangle=\left|\Omega_{t}^{+}\right\rangle+\frac{\varepsilon}{i} U_{+}(t)\left|\Omega_{+}\right\rangle, \\
& \left|\Delta_{t}^{-}\right\rangle=\left|\Omega_{t}^{-}\right\rangle+\frac{\varepsilon}{i} U_{-}(t)\left|\Omega_{-}\right\rangle
\end{aligned}
$$

and $\left|\Omega_{ \pm}\right\rangle=\int_{0}^{t} d t^{\prime} U_{ \pm}^{\dagger}\left(t^{\prime}\right) U_{\mp}\left(t^{\prime}\right)|\Omega\rangle$. The effect of dissipation on the Cheshire Cat paradox is again governed by a quantity

$$
Q_{ \pm}^{\varepsilon}=\left\langle\Omega \mid \Delta_{t}^{+}\right\rangle \pm\left\langle\Omega \mid \Delta_{t}^{-}\right\rangle
$$

which is modified only quantitatively with respect to $Q_{ \pm}$in (29). There are two types of matrix elements (scalar products) in (35). The first is $\left\langle\Omega \mid \Omega_{t}^{ \pm}\right\rangle$already calculated in the previous section in (20); the second is expressed as a time integral with $T=t^{\prime}-t$ :

$$
\left\langle\Omega \mid U_{ \pm}(t) \Omega_{ \pm}\right\rangle=\int_{0}^{t} d t^{\prime}\left\langle\Omega_{T}^{ \pm} \mid \Omega_{t^{\prime}}^{\mp}\right\rangle,
$$


where, for the assumed symmetric coupling $g_{+}=g_{-}$, the integrand $\left\langle\Omega_{T}^{ \pm} \mid \Omega_{t^{\prime}}^{\mp}\right\rangle=e^{i v} \Upsilon$ with $v=K\left(t^{\prime}\right)-$ $K(T)$ and $\Upsilon=\exp \left[\int_{0}^{\infty} d \omega\left(g^{2}(\omega) / h^{2}(\omega)\right)\left(\Upsilon_{R}+i \Upsilon_{I}\right)\right], \Upsilon_{R}=$ $-3+2 \cos (h(\omega) T)+2 \cos \left(h(\omega) t^{\prime}\right)-\cos (h(\omega) t)$, and $\Upsilon_{I}=$ $\sin (h(\omega) T)+\sin (h(\omega) t)-\sin \left(h(\omega) t^{\prime}\right)$. Although the time integral in (36) requires numerical evaluation, one concludes that a (small) dissipation rate impacts, according to (35), only qualitatively the "paradoxial" properties of the Cheshire Cat [3] as it was the case for a (symmetric) pure decoherence (cf. (20)). It supports the claim that the Cheshire Cat paradox remains surprisingly robust against realistic decoherence sources. Again, as it was in the pure dephasing case, the most influential part is a real part of (35) and the only result of dissipation is to speed up its time decay. Roughly speaking, the presence of weak (perturbative) dissipation, despite its qualitative essential difference, is qualitatively similar to decreasing $\mu$ for pure dephasing, that is, to approaching Ohmic limit in Figure 1.

There is no generally valid model for reduced dynamics of open quantum systems [10]. The rigorous weak coupling scheme, the Davies approach $[24,28]$, works well for long time of evolution. Its applicability for short time horizons may be questioned [28]. However, the Cheshire Cat experiment is performed in a relatively short time scale related to a passage of photos via an interferometer [6] and the Davies approximation is not optimal. The approach proposed in this section is, one can say, on the opposite side of the scale; it is valid for time scales which are short in comparison with other time scales in the system and hence it is more suitable for realistic modelling of the dissipative Cheshire Cat effect.

\section{Summary}

There is an unavoidable influence of an environment on any experimental investigation of the quantum Cheshire Cat behaviour [6]. In our work, we analyzed the fundamental Mach-Zehnder-like interferometric system where the Cheshire Cat effect occurs [3]. We studied this "paradox" in the presence of decoherence caused by an infinite bosonic environment. We assumed that bosonic system couples solely to photonic polarization in only one of two arms of the interferometer; that is, either the path where the cat resides or the one with its grin was assumed "noisy" modifying the preselected state. The most general conclusion of our modelling is that the decoherence affects, one can say "attracts," the cat if it is present in the path occupied by the grin but "attracts" the grin if it is present in the path where the cat resides.

Further we limited our consideration to probably simplest models of pure decoherence (dephasing) and weak dissipation. In return, we gain analytic results for weak values of cat's position and its grin. We showed that the Cheshire Cat effect is preserved in the presence of pure decoherence provided that the decoherence is symmetric. Such a decoherence modifies quantitatively only the grin of the cat in (27). However, a position of the cat remains not influenced by symmetric pure decoherence (cf. (26)). This situation changes qualitatively if one passes from the symmetric van Hove [22] model to any among asymmetric dephasings [19] including the Friedrichs model [23]. The larger the asymmetry of dephasing is, the more lethal it is for the separation of the position and the grin of the Cheshire Cat.

Pure decoherence, despite its applicability [15], may seem artificial as in real systems there is always an energy dissipation. Unfortunately, there is no general and effective method of handling quantum systems out of thermal equilibrium [24]. An example of one of the most general methods is the weak coupling Davies approach $[24,28]$. This approach allows for effective modelling nonequilibrium thermodynamics of open quantum systems [29] but, due to applied scaling of time [28], is rather suitable for long time scales of quantum dynamics $[24,28]$. In our work, we applied a different approach. Using time-dependent perturbation expansion with respect to $+\leftrightarrow-$ polarization transition parameter $\varepsilon$ in (32), we showed that the predictions of the pure decoherence model hold true also for systems affected by a small rate of dissipation. This result is clearly limited (not only by small values of $\varepsilon$ but also in time) by credibility of the perturbative expansion (cf. [27]).

Quantum paradoxes are usually invoked as witnesses supporting "bizzareness" of quantum mechanics and its problematic applicability in description of macroscopic world. One expects that there are mechanisms limiting "quantumness" of everyday life. Decoherence is a natural candidate for such a mechanism. Nevertheless, recent experimental developments suggest something very different: quantum properties, including the "paradoxes," can survive in the presence of decoherence. Our work is a modest attempt to describe one of spectacular quantum effects, the Cheshire Cat, in the presence of certain class of decoherence mechanisms. We hope that our results can support experimental investigations of quantum phenomena.

\section{Conflicts of Interest}

The authors declare that there are no conflicts of interest regarding the publication of this paper.

\section{Acknowledgments}

This work has been supported by the NCN Grant 2015/19/B/ST2/02856.

\section{References}

[1] Y. Aharonov and D. Rohrlich, Quantum Paradoxes, Wiley, 2007.

[2] M. Schlosshauer, Decoherence and the quantum-to-classical transition, Springer, 2007.

[3] Y. Aharonov, S. Popescu, D. Rohrlich, and P. Skrzypczyk, "Quantum Cheshire Cats," New Journal of Physics, vol. 15, 2013, http://stacks.iop.org/1367-2630/15/i=11/a=113015.

[4] R. Corrêa, M. F. Santos, C. H. Monken, and P. L. Saldanha, "'Quantum Cheshire cat' as simple quantum interference," New Journal of Physics, vol. 17, no. May, 2015, http://stacks.iop.org/ $1367-2630 / 17 / \mathrm{i}=5 / \mathrm{a}=05304$.

[5] T. Denkmayr, H. Geppert, S. Sponar et al., "Observation of a quantum Cheshire Cat in a matter-wave interferometer 
experiment," Nature Communications, vol. 5, Article ID 4492, 2014, http://dx.doi.org/10.1038/ncomms5492.

[6] J. M. Ashby, P. D. Schwarz, and M. Schlosshauer, "Observation of the quantum paradox of separation of a single photon from one of its properties," Physical Review A, vol. 94, 2016, http://link.aps.org/doi/10.1103/PhysRevA.94.012102.

[7] J. Dressel, M. Malik, F. M. Miatto, A. N. Jordan, and R. W. Boyd, "Colloquium: Understanding quantum weak values: Basics and applications," Reviews of Modern Physics, vol. 86, no. 307, 2014, http://link.aps.org/doi/10.1103/RevModPhys.86.307.

[8] D. P. Atherton, G. Ranjit, A. A. Geraci, and J. D. Weinstein, "Observation of a classical Cheshire cat in an optical interferometer," OSA Publishing, Optics Letters, vol. 40, pp. 879-881, 2015, http://ol.osa.org/abstract.cfm?URI=ol-40-6-879.

[9] M. Richter, B. Dziewit, and J. Dajka, Frontiers of Quantum and Mesoscopic Thermodynamics, Springer, 2011, URL https, https://fqmt.fzu.cz/17/printables/absbookFQMT17.pdf.

[10] H.-P. Breuer and F. Petruccione, "The Theory of Open Quantum Systems," The Theory of Open Quantum Systems, pp. 1-656, 2010.

[11] L.-M. Chang, L. Liu, Y.-H. Gong, M.-Q. Tan, Y.-D. Yu, and Z.-Y. Li, "Polarization-independent directional coupler and polarization beam splitter based on asymmetric cross-slot waveguides," OSA Publishing, Applied Optics, vol. 57, pp. 678683, 2018, http://ao.osa.org/abstract.cfm?URI=ao-57-4-678.

[12] S. Rojas-Rojas, L. Morales-Inostroza, U. Naether et al., "Analytical model for polarization-dependent light propagation in waveguide arrays and applications," Physical Review A, vol. 90, Article ID 063823, 2014, https://link.aps.org/doi/10.1103/ PhysRevA.90.063823.

[13] L. Aolita, F. de Melo, and L. Davidovich, Reports on Progress in Physics, vol. 78, IOPscience, 2001, http://stacks.iop.org/00344885/78/i=4/a=042001\&lt.

[14] R. Alicki, "Pure decoherence in quantum systems," Open Systems \& Information Dynamics, vol. 11, no. 1, pp. 53-61, 2004.

[15] D. I. Schuster, A. A. Houck, J. A. Schreier et al., "Resolving photon number states in a superconducting circuit," Nature, vol. 445, no. 7127, pp. 515-518, 2007.

[16] J. Dajka and J. Łuczka, "Origination and survival of qudit-qudit entanglement in open systems," Physical Review A: Atomic, Molecular and Optical Physics, vol. 77, no. 6, 062303, 7 pages, 2008.

[17] O. Bratteli and D. W. Robinson, Operator Algebras and Quantum Statistical Mechanics, Springer, Berlin, Germany, 2nd edition, 1997.

[18] A. M. Perelomov, Generalized Coherent States and Their Applications, Texts and Monographs in Physics, Springer, Berlin, Germany, 1986.

[19] J. Dajka, M. Mierzejewski, and J. Łuczka, "Fidelity of asymmetric dephasing channels," Physical Review A, vol. 79, no. 1, 2009, http://link.aps.org/doi/10.1103/PhysRevA.79.012104.

[20] J. Dajka and J. Łuczka, "Bifurcations of the geometric phase of a qubit asymmetrically coupled to the environment," Journal of Physics A: Mathematical and General, vol. 41, no. 44, Article ID 442001, 44001, 8 pages, 2008.

[21] K. Roszak and P. MacHnikowski, "Erratum: Complete disentanglement by partial pure dephasing (Physical Review A (2006) 73 (022313))," Physical Review A: Atomic, Molecular and Optical Physics, vol. 73, no. 2, Article ID 029906, 2006.

[22] L. van Hove, "Les difficultés de divergences pour un modèle particulier de champ quantifié," Physica A: Statistical Mechanics and its Applications, vol. 18, no. 3, pp. 145-159, 1952.
[23] K. O. Friedrichs, "On the perturbation of continuous spectra," Communications on Pure and Applied Mathematics, vol. 1, no. 4, pp. 361-406, 1948.

[24] R. Alicki and K. Lendi, Quantum dynamical semigroups and applications, vol. 717 of Lecture Notes in Physics, Springer, Berlin, Germany, Second edition, 2007.

[25] M. Łobejko, M. Mierzejewski, and J. Dajka, "Interference of qubits in pure dephasing and almost pure dephasing environments," Journal of Physics A: Mathematical and General, vol. 48, no. 27, 2015.

[26] R. S. Phillips, "Perturbation theory for semi-groups of linear operators," Transactions of the American Mathematical Society, vol. 74, pp. 199-221, 1953.

[27] T. Kato, Perturbation Theory for Linear Operators, vol. 132 of Classics in Mathematics, Springer, Berlin, Germany, 1995.

[28] E. B. Davies, Quantum Theory of Open Systems, Academic Press (Harcourt Brace Jovanovich, Publishers), London, UK, 1976.

[29] H. Spohn and J. L. Lebowitz, "Irreversible Thermodynamics for Quantum Systems Weakly Coupled to Thermal Reservoirs," in Advances in Chemical Physics: For Ilya Prigogine, A. S. Rice, Ed., pp. 109-142, John Wiley \& Sons, Inc, 2007, http://dx.doi.org/10.1002/9780470142578.ch2. 


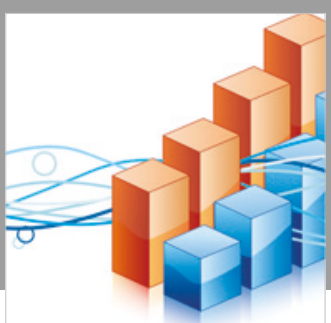

Advances in

Operations Research

\section{-n-m}
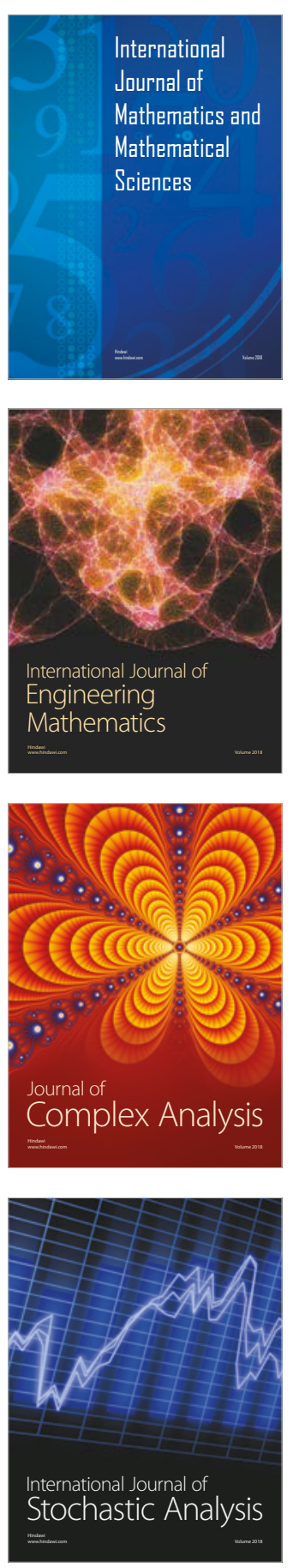
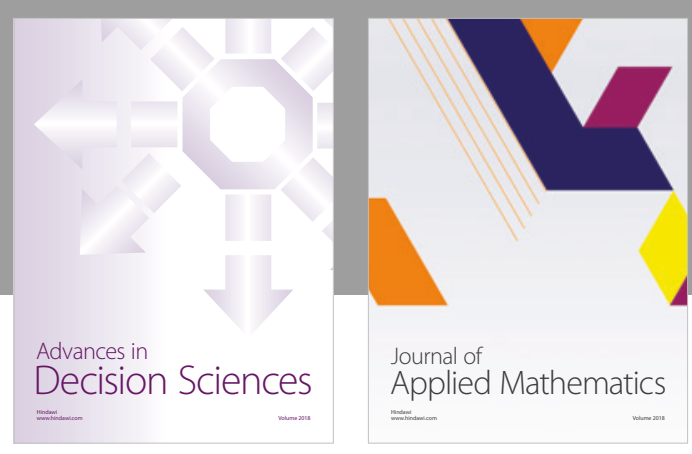

Journal of

Applied Mathematics
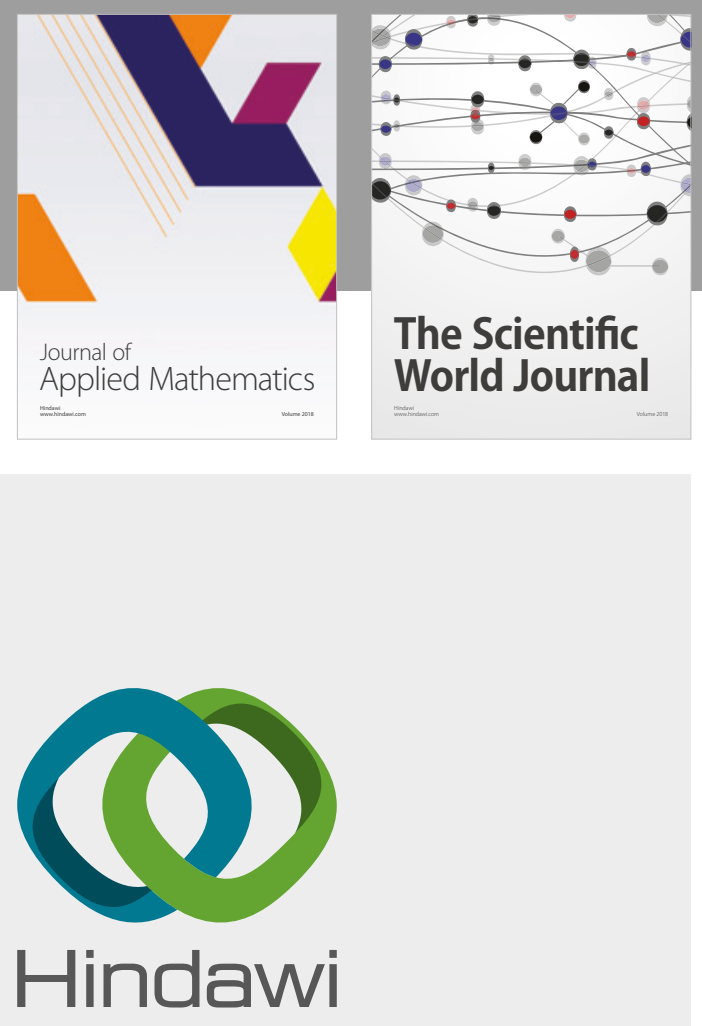

Submit your manuscripts at

www.hindawi.com

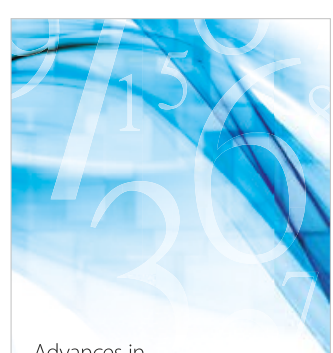

Advances in
Numerical Analysis
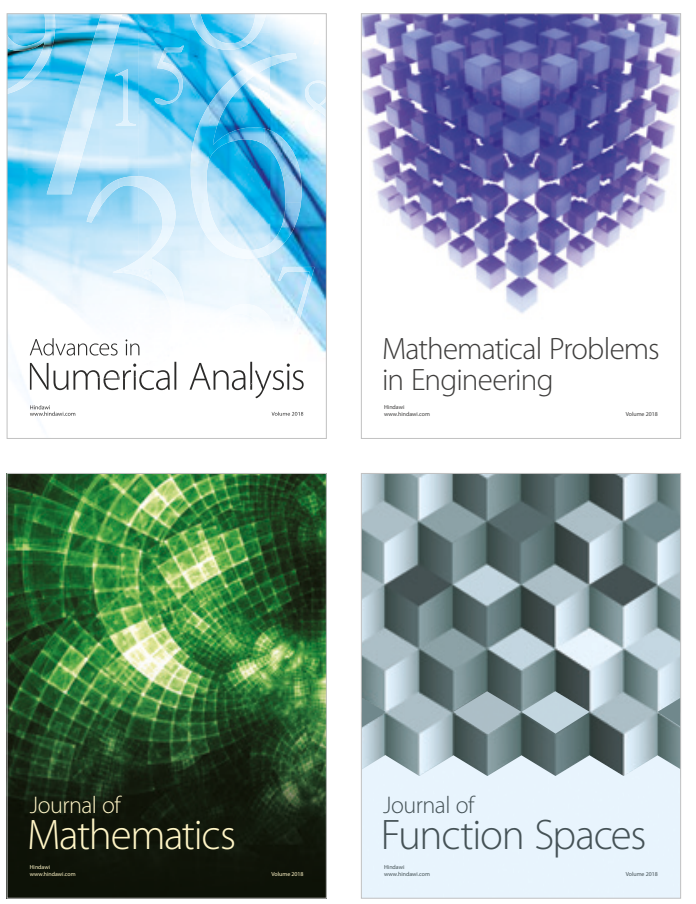

Mathematical Problems in Engineering

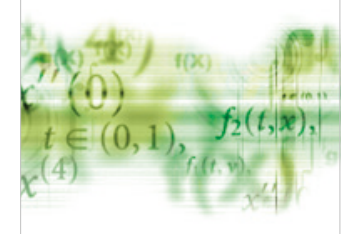

International Journal of

Differential Equations

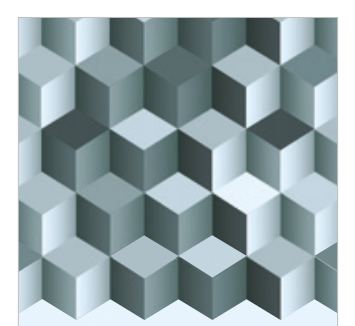

Journal of

Function Spaces

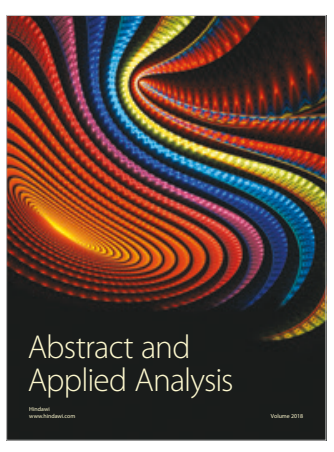

The Scientific

World Journal

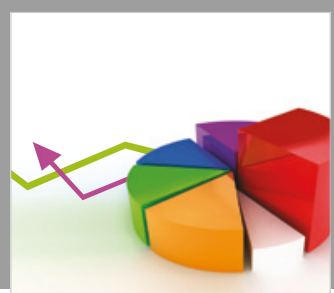

Journal of

Probability and Statistics
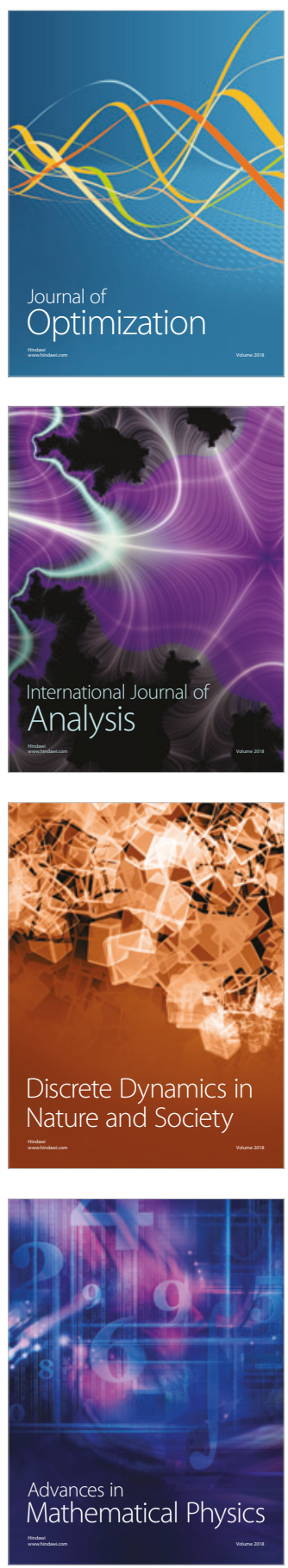